\title{
El papel de la inteligencia colectiva en logistica y su impacto en la eficiencia
}

\author{
El papel de la inteligencia colectiva en logistica y su impacto en la eficiencia
}

DOI: $10.46932 / \operatorname{sfjdv2n2-141}$

Received in: March 1st, 2021

Accepted in: May 30th, 2021

MGTI. Hermes Orestes Cedillo-Campos

Universidad Autónoma de Querétaro, Cerro de Las Campanas, s/n, Querétaro,México.

E-mail: hermesorestescc@gmail.com

Dr. Juan José Méndez-Palacios

Universidad Autónoma de Querétaro, Cerro de Las Campanas, s/n, Querétaro,México. E-mail: juanjo@uaq.mx

\begin{abstract}
RESUMEN
Se expone una revisión crítica de la literatura al cruce de la inteligencia colectiva y cadena logística con base en el análisis de setenta y nueve artículos publicados entre el año 2000 y 2020. Además, se analiza la postura que abordan diferentes autores tanto con respecto a la importancia de integrar todos los niveles de la cadena logística, como en cuanto a las diversas formas del conocimiento colectivo. El objetivo del artículo es analizar con base en la literatura existente, las actuales formas organizacionales en inteligencia colectiva, pero buscando identificar la arquitectura del conocimiento para potencializar la eficiencia logística. Para ello se estudió las formas de interacción entre las organizaciones reportadas por los autores, con el fin de identificar los procesos, servicios y/o actividades comunes a diferentes cadenas logísticas. De este modo, se identificaron dos aspectos importantes. Por un lado, la formación de conocimiento colectivo es un factor crítico que incide en la generación de oportunidades competitivas que, permite crear base de desarrollo y crecimiento para las organizaciones en cuestión de innovación. Por otro lado, se pudo establecer que la inteligencia colectiva se ha convertido en un paradigma para las organizaciones industriales que, favorece la eficiencia logística de distintos sectores. Como resultado, se identificaron variables fundamentales para el desarrollo de un modelo analítico que busca determinar los procesos de la cadena logística.
\end{abstract}

Palabras Clave: Inteligencia colectiva en logistica, Eficiencia logística, Gestion del conocimiento, Competitividad, Cadena logistica.

\begin{abstract}
A critical review of the literature on the intersection of collective intelligence and logistics chain is presented based on the analysis of seventy-nine articles published between 2000 and 2020. In addition, the position taken by different authors regarding the importance of integrating all levels of the logistics chain, as well as the various forms of collective knowledge, is analyzed. The objective of the article is to analyze, based on the existing literature, the current organizational forms of collective intelligence, but seeking to identify the architecture of knowledge to enhance logistics efficiency. To this end, the forms of interaction between organizations reported by the authors were studied in order to identify the processes, services and/or activities common to different logistics chains. Two important aspects were identified. On the one hand, the formation of collective knowledge is a critical factor that influences the generation of competitive opportunities that create a basis for the development and growth of organizations in terms of innovation. On the other hand, it was established that collective intelligence has
\end{abstract}


become a paradigm for industrial organizations that favors logistical efficiency in different sectors. As a result, fundamental variables were identified for the development of an analytical model that seeks to determine the processes of the logistics chain.

Keywords: Collective intelligence in logistics, Logistics efficiency, Knowledge management, Competitiveness, Logistics chain.

\section{INTRODUCCION}

Una red con mecanismos avanzados de transferencia de conocimiento será capaz, de competir más eficazmente con otras redes de producción, con secuencias de intercambio de conocimientos menos efectivas en términos de innovación (von Hippel, 1986; Dyer and Singh, 1998). Si bien una rápida búsqueda en Google Académico puede arrojar más de 28,400 de referencias al teclear la palabra "Inteligencia colectiva en Logística" Derivado de la evolución de las industrias, desde la operación intensiva de procesamiento de datos hasta las operaciones basadas en la información y las empresas basadas en el conocimiento (Liew, 2008), existe el deseo de comprender la inteligencia colectiva (IC). Este llamado se hace evidente en el contexto de la cadena logística (CL), ya que la (CL) puede verse como una cadena sistémica compleja en donde planifica y controla los bienes de la organización, en donde abarca el flujo de capital impulsado por la información y conocimiento.

El ideal de la inteligencia colectiva, de acuerdo con (Levy, 1997), considera la inteligencia valorada desde la economía, lo jurídico, lo humano y lo técnico para impulsar las competencias de la persona.

De manera exógena a las empresas, y en general a las instituciones, la inteligencia colectiva ha tenido un fuerte impulso gracias al advenimiento de internet, y en el último lustro a las comunidades virtuales, fusionando lo cognitivo con lo tecnológico (Levy, 1997; Valdés, 2010). Desde el área de la pedagogía hasta la ingeniería. Nuestro análisis para la (IC) ha emergido con fuerza el concepto de inteligencia colectiva, entendida como: "grupos de individuos actuando colectivamente en formas que parecen inteligentes" (Malone et al., 2009).

Pierre Levy (1997) señala que la inteligencia alimenta el poder cognitivo, de percepción, acción, planeación y coordinación que le otorga al ser vivo una memoria, imaginación y generar nuevos conocimientos.

El presente artículo se encuentra organizado de la siguiente forma. En la sección II se muestra la metodología que se empleó para recolectar, analizar y clasificar la información. En la sección III los resultados y discusión derivada del análisis de la información sobre inteligencia colectiva y la cadena logística son presentados. En la sección IV se exponen las conclusiones de la investigación, así como 
algunas líneas futuras de investigación.

\section{METODOLOGIA}

El método de análisis seleccionado para este artículo fue de tipo cualitativo y se dividió en dos pasos: 1. Recolección de la información; y 2. Clasificación de la misma. Como resultado, se desarrolló un análisis crítico de la literatura a la intersección de inteligencia colectiva y cadena logística.

A. Recolección de la información.

Para la colecta y análisis de la información, se estableció el periodo de tiempo entre el año 2000 a junio 2020, teniendo como palabras claves de búsqueda "inteligencia colectiva" y "cadena logística". Estas palabras fueron buscadas en bases de datos como: Emerald Insight (http://www.emeraldinsight.com/), ScienceDirect (http://www.sciencedirect.com/) y $\quad$ Wiley

Online Libraly (http://www.onlinelibrary.wiley.com/). Mediante el uso de la herramienta de búsqueda avanzada, con el uso del contenedor "Y", se especificó que las palabras clave se conectaran entre sí. Principalmente dentro del resumen, título y palabras clave.

Como resultado, se obtuvieron setenta y nueve (79) artículos entre ellos: casos de estudio, artículos de investigación, capítulos de libros, etc.

Todos ellos se descargaron para un análisis detallado y con ello, seleccionar aquellos que se relacionaran o que tuvieran mayor relevancia con el tema de inteligencia colectiva y cadena logística. Se buscó información provista por los autores sobre desarrollos, actividades o servicios que fuera aptos de ser agrupados y además, establecieran variantes para el proceso de un posible modelo analítico para mejorar la eficiencia logística en inteligencia colectiva.

Fig. 1 Artículos por base de datos 


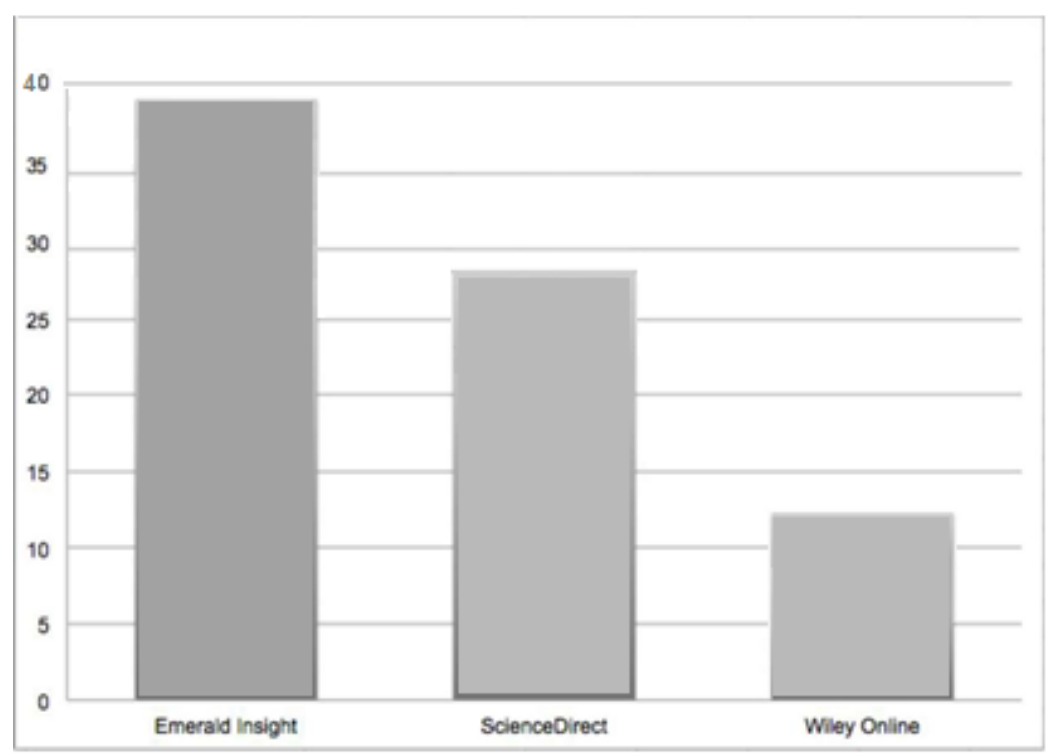

De los artículos recolectados, primero se cuantifico la cantidad de artículosobtenidos por base de datos (ver figura 1 ).

1). De "Emerald Insight" se obtuvo la mayor cantidad de artículos con un total de veintiocho (38), de "ScienceDirect" se obtuvieron veinticinco (29) y "Wiley Online Library" solo nueve (12) artículos.

Por otra parte, también se analizó el crecimiento de las publicaciones a lo largo del periodo de estudio. Como resultado, se estableció el crecimiento del estudio del tema. El año 2020 mostro ser el de mayor productividad con diez (10) artículos publicados, por su grado de beneficio el tema ha aumentado el interés entre los investigadores (ver figura 2). 
Fig. 2 Publicación de artículos por año durante el periodo 2000 - 2020

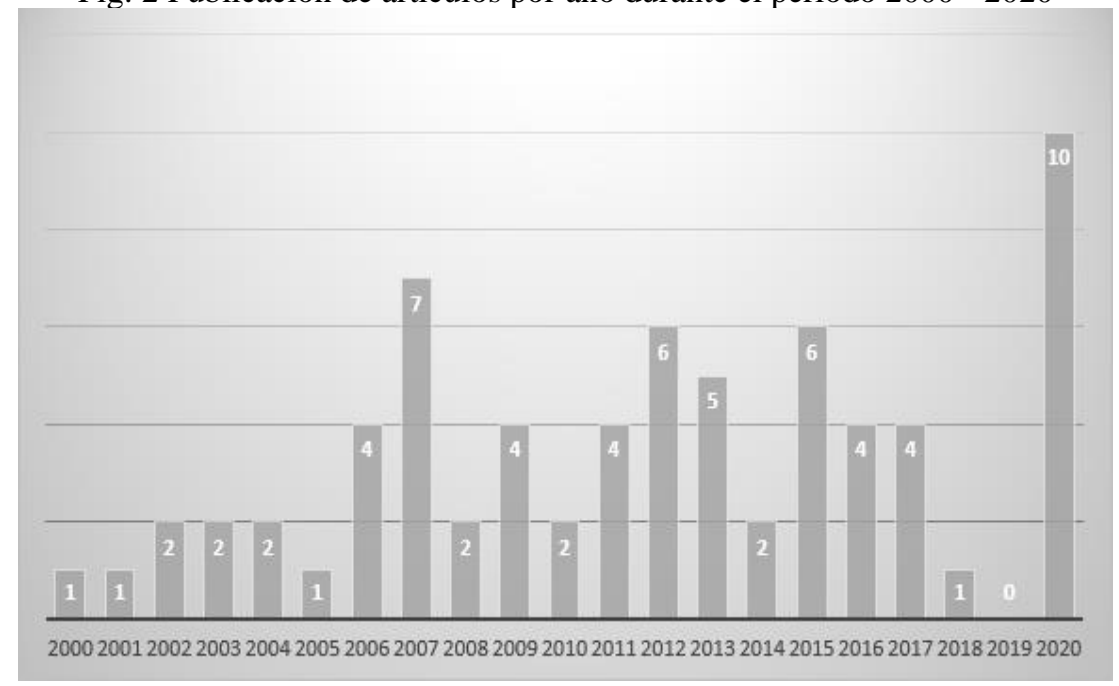

B. Clasificación de la información.

Para la selección de los artículos que se incluyeron dentro de nuestro estudio, primero se leyó el resumen de cada uno de los artículos. Se seleccionaron los artículos que exponían información sobre temas como: conocimiento colectivo, gestión del conocimiento, ingeniería de conocimiento, capital intelectual, redes colectivas, innovación, conocimiento, fuente de conocimiento y empresa, actividades logísticas. Con base a lo anterior se seleccionó un total de diez (10) artículos, más tres (3) artículos que se tomaron como referencia debido a que la mayoría de los artículos hacían referencia a ello.

Para la clasificación de la información recolectada, se requirió organizarla de forma que se obtuviera de cada uno de los artículos los aspectos más relevantes. Para esto se utilizó una hoja de cálculo que contenía los siguientes campos:

- $\quad$ Nombre del articulo

- $\quad$ Autores

- $\quad$ Fecha de publicación

- Teorías o formas de organización analizadas

- Características de la forma de organización abalizada

- Ventajas y desventajas del enfoque propuesto.

- $\quad$ Desarrollo y/o aplicación de un modelo.

- Variables identificadas / utilizadas

- Resultados y conclusiones relevantes. 


\section{RESULTADOS Y DISCUSIÓN}

Derivado de los análisis desarrollados, se identificaron tres vertientes sobre la evaluación de las interacciones dentro de la logística. Estas vertientes identificadas son: i) Gestión del conocimiento; ii) De los datos al conocimiento organizacional; iii) inteligencia colectiva en las organizaciones logísticas.

\section{A. Gestión del conocimiento.}

Para toda organización logística el elemento más importante es el capital humano; se debe de comprender como el origen de un ambiente que propicia el desarrollo de una cultura organizacional como único modo de poder difundir el conocimiento a todos los miembros de la organización.

Una organización inteligente es la que es capaz de recibir y procesar la información, crear conocimiento con ella y usarlo para la toma de decisiones de manera eficaz. Esencialmente el objetivo de la gestión del conocimiento es el manejo sistemático del conocimiento y el conocimiento potencial (Heisig, 2009) como definen Chuang, Liao y Lin (2013): "un proceso específico, sistémico y organizacional, para crear, transferir, integrar y aprovechar el conocimiento asociado, que el conocimiento de una unidad funcional particular se aplica a otras unidades funcionales que difieren en la ventaja competitiva". La gestión del conocimiento también implica el desarrollo de un conjunto de capacidades organizacionales que permitirían a la empresa reconocer, crear, transformar y distribuir el conocimiento (Gold , Malhotra y Segars, 2001) con un enfoque en el flujo de conocimiento (Alavi y Leidner, 2001) y la creación de valor a partir de los recursos intangibles dentro y fuera de una organización (RubensteinMontano, Liebowitz, Buchwalter, McCaw, Newman \& Rebeck, 2001). Por su parte, (Liew, 2008) reconoció la gestión del conocimiento como "una de las principales fuerzas impulsoras del cambio organizacional y la creación de valor desde principios de 1990", que se ha vuelto más complejo como resultado de una evolución del concepto de gestión.

\section{B. De los datos al conocimiento organizacional.}

Por tratarse de un campo de estudio de reciente incorporación a las ciencias de la administración, los conceptos asociados y la terminología relacionada aún se encuentran en su fase de desacuerdo por parte de los investigadores y expertos (De luna, 2008). Las definiciones más significativas para los autores principales de estos términos son: (Sanguino R., 2003).

- Dato: Elemento o cantidad conocida que sirve de base para la resolución de un problema. Principio en el que se asienta una discusión. Elemento o base para la formulación de un juicio. Lo que se presenta a la conciencia como inmediato, no construido o elaborado. Elemento de información o representación de 
hechos o de instrucciones, en forma apropiada para el almacenamiento, procesamiento o transmisión por medios automáticos.

- Información: Acto o efecto de informar(se), informe. Dato acerca de alguien o de algo. Comunicación o noticia traída al conocimiento de una persona o del público. Instrucciones, dirección. Conocimiento amplio y bien fundamentado, resultante del análisis y combinación de varios informes. Según la teoría de la información, medida de reducción de error, sobre un determinado estado de cosas, por intermedio de un mensaje (en este sentido, información no debe ser confundida con significado y presentarse como función directa del grado de funcionalidad, imprevisibilidad y valor sorpresa del mensaje, siendo cuantificada en bits de información).

- Conocimiento: Acto o efecto de conocer. Idea, noción. Noticias, ciencia. Practica de vida, experiencia. Discernimiento, criterio, apreciación. Conciencia de sí mismo, acuerdo. En el sentido más amplio, atributo general que tiene los seres vivos de regir activamente el mundo circulante, en la medida de su organización biológica y en el sentido de su supervivencia.

\section{Inteligencia colectiva en las organizaciones logísticas.}

En una economía industrial basada en el conocimiento, la logística desempeña un papel estratégico cada vez más importante para las organizaciones que se esfuerzan por seguir el ritmo de los cambios del mercado y la integración de la cadena de suministro (Mahesh S. Raisinghani, Laura L. Meade, 2005). La inteligencia colectiva ha sido un tema importante para académicos y profesionales colectivamente por igual en la década de los 1990. Crear inteligencia colectiva implica apoyar a que todo el sistema madure simultáneamente, es decir, adquiera destreza no solo en lo individual, si no en lo colectivo, para encontrar soluciones efectivas.

Son muchos los estudios que demuestran el valor del bienestar en el trabajo y como las personas que son más felices son también más productivas (Lewis, 2011). Es por ello que el desafío hoy no está en demostrar el valor del clima laboral, sino el cómo cambiar la mirada que se tiene de este y su relación con la gestión y la forma de llevar adelante las actividades que generan los resultados (Vergara, 2015).

\section{CONCLUSIONES}

El presente artículo analiza con base en la literatura existente, las actuales formas de organización en la aplicación del conocimiento colectivo buscando identificar la constitución de la inteligencia colectiva en logística para eficientar la cadena logística. Para ello se estudiaron las formas de interacción entre las organizaciones reportadas por los autores, de forma que fuera posible identificar los procesos, 
organización y/o actividades diferentes en la cadena logística y que pudieran agregar valor al conocimiento colectivo.

El presente estudio contribuye al cuerpo del conocimiento a través de un análisis que favorece la comprensión de los nuevos paradigmas operacionales subyacentes en la nueva economía del conocimiento. Lo anterior permitió crear una base sólida que se puede tomar como base para el desarrollo de indicadores que puedan mostrar no solo el desempeño de la inteligencia colectiva en logística y su interacción, sino también para nuevos patrones de correlación entre variables clave. 


\section{REFERENCIAS}

Alavi, M., \& Leidner, D.E. (2001). Review: Knowledge Management and KnowledgeManagement Systems: Conceptual Foundations and Research Issues. MIS Quarterly, 25(1), 107-136. https://doi.org/10.2307/3250961

Chuang, S.H., Liao, C., \& Lin, S. (2013). Determinants of knowledge management with information technology support impact on firm performance. Information Technology and Management, 14(3), 217230. https://doi.org/10.1007/s10799-013-0153-1

De Luna Noyola, A. G. (2008). Capital humano: Gestion por competencias laboralesen la administracion publica. pp. 187-192 (reimp. 2011)

Dyer, J.H. and Singh, H. (1998), "The relational view: cooperative strategy and sources of interorgani advantage", Academy of Management Review, Vol. 23 No. 4, pp. 660-79

Gold, A.H., Malhotra, A., \& Segars, A.H. (2001). Knowledge management: an organizational capabilities perspective. Journal of Management Information Systems, 18(1), 688-698. https://doi.org/10.1080/07421222.2001.11045669

Heisig, P. (2009). Harmonisation of knowledge management comparing $1 \underline{60} \mathrm{KM}$ frameworks around the globe. Journal of Knowledge Management, 13(4), 4- 31. https://doi.org/10.1108/13673270910971798

Levy, P. (1997). Collective intelligence: mankind's emerging world in cyberspace. New York, NY, EUA: Plenum.

Lewis Sarah (2011). Positive Psychology at Work. San Francisco, CA: Wiley Blackwell \& Sons.

Liew, C.B.A. (2008). Strategic integration of knowledge management and customer relationship management. Journal of Knowledge Management, 12(4), 131-146. https://doi.org/10.1108/13673270810884309

Mahesh S. Raisinghani, Laura L. Meade, (2005) "Strategic decisions in supply chain intelligence using knowledge management: an analytic network process framework", Supply Chain Management: An International Journal, Vol. 10 Issue: 2, pp.114-121, https://doi.org/10.1108/13598540510589188

Malone. T., Laubacher, R., and Dellarocas, C. (2009). Harnessing Crowds: Mappingthe Genome of Collective Intelligence. MIT Center for Collective Intelligence, Massachusetts Institute of Technology, Cambridge, MA.

Rubenstein-Montano, B., Liebowitz, J., Buchwalter, J., McCaw, D., Newman, B., \& Rebeck, K. (2001). A systems thinking framework for knowledge management. Decision Support Systems, 31(1), 5-16. https://doi.org/10.1016/S0167-9236(00)00116-0

Sanguino, R. (2003): "La Gestión del conocimiento. Su importancia como recurso estratégico para la organización", [en línea] 5campus.org, Marketing

<http://www.5campus.org/leccion/km> [22 de diciembre del 2017]

Valdés Godínez, J. C. (2009). Los escenarios pedagógicos tecnoculturales y su importancia en la conformación de formas de razonamiento. Tesis para obtener el grado de doctor, Universidad Nacional 


\section{Autónoma de México, México DF}

Vergara Venegas, S. (2015), Construir inteligencia colectiva en la organización: unanueva manera de entender y gestionar el clima laboral para alinear el bienestarde las personas con la gestión de la empresa. CIP Pontificia Universidad Católica de Chile.

Von Hippel, E. (1986), "Lead users: a source of novel product concepts", Management Science, Vol. 32 No. 7, pp. 791-805. 